a DSA-10 field of view

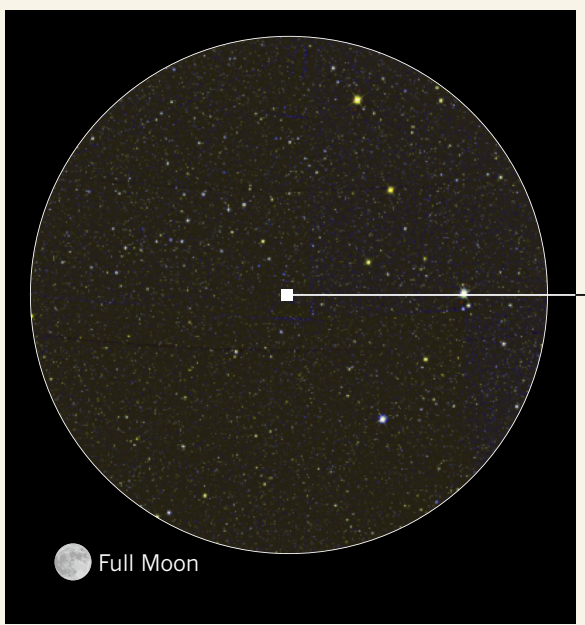

b FRB location and likely host galaxy

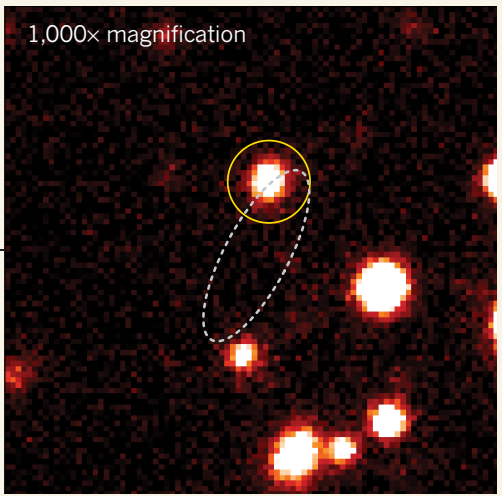

Figure 1 | Localization of a fast radio burst (FRB). a, Ravi et al. ${ }^{3}$ report observations from an array of radio telescopes known as the Deep Synoptic Array 10-antenna prototype (DSA-10). The field of view of DSA-10 is roughly 40 square degrees ${ }^{7}$, which is about 200 times the area on the sky that is covered by the full Moon when viewed from Earth's surface. $\mathbf{b}$, Ravi and colleagues used DSA-10 to precisely determine the position of an FRB - a millisecond-duration flash of radio waves. The broken white ellipse shows the region in which the FRB could be located. The authors then identified a massive galaxy (indicated by the yellow circle) that is the likely host of the FRB.

host galaxy is markedly different from the host ${ }^{5}$ of the well-localized source of the repeating FRB. It is 1,000 times more massive, and shows none of the prodigious star formation that is associated with the environment of the repeating-FRB source. Only a week before Ravi and colleagues' work was published online, a similar breakthrough was reported ${ }^{9}$ using the Australian Square Kilometre Array Pathfinder (ASKAP) telescope. The authors of that paper achieved an even more precise localization of another one-off FRB, and also demonstrated that it originates from a massive galaxy that shows little signs of active star formation.

So, do these results mean that one-off FRBs and repeaters come from different galaxy types, and that they have physically different origins? Do astronomers have two puzzles on their hands? Perhaps, but with only three FRB host galaxies identified so far, many alternatives remain open. For instance, it is possible that all FRBs are generated by hyper-magnetized neutron stars, but that there are various ways in which such neutron stars can be produced $^{10}$. Some might form directly through the collapse of a massive star, whereas others might be made from old neutron stars in a binary system that smash into each other as the orbital distance between them decreases. This difference could explain why some FRBs seem to originate from star-forming regions and others do not ${ }^{10}$.

Excitingly, we will soon know a lot more. The mystery of FRBs has driven many teams worldwide to tune radio telescopes towards discovering and localizing these signals, and many thousands of FRBs are thought to happen somewhere on the sky each day ${ }^{2}$. The fact that fewer than 100 FRB sources have been detected is a reflection of the small fields of view of existing radio telescopes. If a sensitive radio telescope could be built that has a continuous view of the entire sky, FRBs would look like a fireworks display. However, wide-field telescopes such as the Canadian Hydrogen Intensity Mapping Experiment ${ }^{11}$ (CHIME) are starting to change the game. It might not be long before astronomers have catalogued thousands of FRB sources and pinpointed at least dozens of them.
The precise localizations from DSA-10 and

\section{TUMOUR BIOLOGY}

\section{YUNPENG LIU \& MICHAEL T. HEMANN}

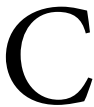
hemotherapy usually works by inducing DNA damage that leads to cell death. However, rather than dying after chemotherapy, some tumour cells enter an inactive state, termed senescence, in which they are alive but have permanently stopped dividing ${ }^{1}$. Although senescence in normal cells drives ageing and tissue degeneration ${ }^{2}$, cancertherapy-induced senescence is associated with
ASKAP are shedding light on the origins of FRBs, but they are also teaching us about the potential use of these signals as astronomical probes. FRBs are delayed in their arrival at Earth by the otherwise invisible material between galaxies ${ }^{2}$. By measuring the magnitude of this time delay, and comparing this measurement with the distance to the host galaxy, astronomers can map the density of ionized material in intergalactic space and thereby weigh the Universe in a unique way. The localizations of one-off FRBs suggest that FRB host galaxies will only slightly skew such measurements. Moreover, the results indicate that, with the detection and localization of thousands of FRBs, a 3D map of the material between galaxies could be made.

Jason Hessels is at ASTRON (the Netherlands Institute for Radio Astronomy) and the Anton Pannekoek Institute for Astronomy, University of Amsterdam, 1098XH Amsterdam, the Netherlands.

e-mail:j.w.t.hessels@uva.nl

1. Lorimer, D. R., Bailes, M., McLaughlin, M. A Narkevic, D. J. \& Crawford, F. Science 318, 777-780 (2007).

2. Petroff, E., Hessels, J. W. T. \& Lorimer, D. R. Astron Astrophys. Rev. 27, 4 (2019).

3. Ravi, V. et al. Nature 572, 352-354 (2019).

4. Spitler, L. G. et al. Nature 531, 202-205 (2016)

5. Bassa, C. G. et al. Astrophys. J. 843, L8 (2017).

6. Margalit, B. \& Metzger, B. D. Astrophys. J. 868, L4 (2018).

7. Kocz, J. et al. Preprint at https://arxiv.org/ abs/1906.08699 (2019).

8. Eftekhari, T. \& Berger, E. Astrophys. J. 849, 162 (2017).

9. Bannister, K. W. et al. Science https://doi. org/10.1126/science.aaw5903 (2019).

10. Margalit, B., Berger, E. \& Metzger, B. D. Preprint at https://arxiv.org/abs/1907.00016 (2019)

11. The CHIME/FRB Collaboration. Nature 566, 230-234 (2019).

\title{
A dynamic view of chemotherapy
}

Chemotherapy can halt cancer by causing cells to enter a non-dividing state called senescence, but sometimes it causes tumour cells to proliferate. It now seems that the dynamics of the protein 21 governs which of these fates occurs.

positive clinical outcomes ${ }^{3}$. Understanding the factors that drive the senescence of tumour cells might thus aid the development of new anticancer treatments. Writing in Cell, Hsu et al. ${ }^{4}$ shed light on a previously unknown aspect of how chemotherapy-induced entry into senescence is controlled.

Although much progress has been made in uncovering factors that drive senescence, the processes that ultimately commit cells to this fate are poorly understood. A growing body 


\section{a Chemotherapy before DNA replication}

High.

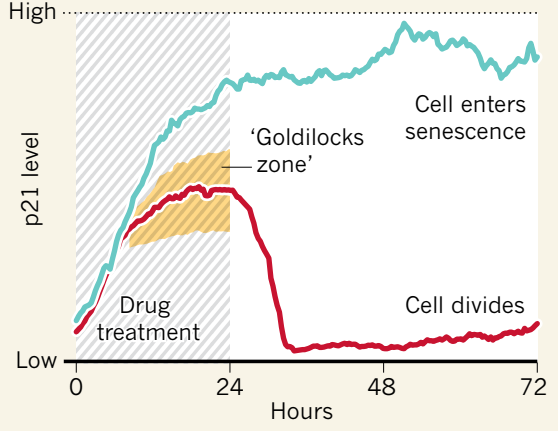

b Chemotherapy during or after DNA replication

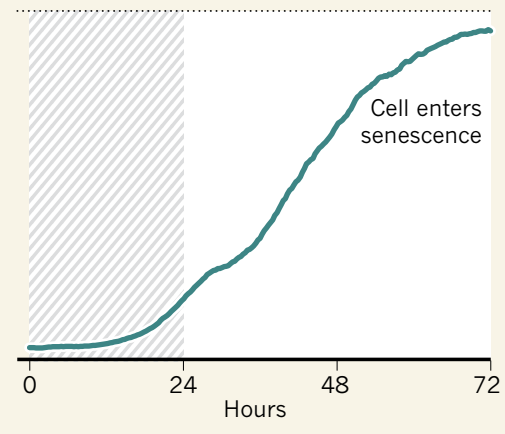

Figure 1 | Levels of p21 protein and cancer-cell fate after chemotherapy. a, Hsu et al. ${ }^{4}$ found that, when human cancer cells grown in vitro were treated with chemotherapy drugs at a stage in the cell cycle before DNA replication, two types of cell fate were observed. In some cells, p21 levels rose and the cells entered a permanent state of non-division termed senescence. In other cells, after an initial rise in the level of $\mathrm{p} 21$, the protein returned to a low level and the cells divided. The authors describe this $\mathrm{p} 21$-dependent switch in cell fate as being affected by a 'Goldilocks zone' (yellow), in which the levels and dynamics of the protein after drug treatment must be 'just right' for cells to be able to halt the cell cycle, repair DNA and then continue to divide. $\mathbf{b}$, By contrast, if cells received drug treatment during or after DNA replication, p21 levels gradually rose and cells entered senescence. (Graphs based on Fig. 3 of ref. 4, showing just the 72-hour window during and after drug treatment.)

of evidence indicates that the regulation of commitment to enter senescence is complex. The mere presence of factors associated with triggering this cell fate is not in itself sufficient to provide an 'on switch' for senescence.

The protein $\mathrm{p} 21$ is probably best known for its role in blocking cell division by inhibiting protein complexes called cyclin-dependent kinases. If DNA damage occurs, p21 activity halts cell division and growth ${ }^{5}$, giving cells time for DNA repair and thereby preventing such damage from having catastrophic cellular consequences. There is evidence that p21 can induce senescence during chemotherapy ${ }^{6}$. Yet, paradoxically, some research suggests that the protein can promote cancer-cell division after chemotherapy ${ }^{7}$. One possible explanation for this discrepancy is that the abundance and dynamics of p21 after chemotherapy have a key role in determining whether cancer cells enter senescence or divide.

To test this idea, Hsu and colleagues developed a microscopy system to study thousands of individual, cultured human lung and colon cancer cells that had been treated with a DNAdamaging chemotherapy drug. The authors monitored the abundance of $\mathrm{p} 21$ by tagging it with a fluorescent protein, and also tracked the progression of the various stages of the cell cycle. In contrast to previous research suggesting that high levels of p21 invariably lead to either cell growth or senescence ${ }^{5,7}$, the authors describe a complex, but unifying, picture of how p21 levels relate to cell fate. Hsu et al. noted that if chemotherapy resulted in an initial rise in p21 levels followed by a decline to low levels, cell division, rather than senescence, occurred (Fig. 1). Cancer cells that entered senescence after drug treatment initially had a low level of p21 that gradually rose to a high level.
Hsu and colleagues suggest that there is a 'Goldilocks zone' for proliferation - a level of p21 that is 'just right' to allow tumour cells to divide after chemotherapy. How might p21 dynamics control cell fate in this way? Chemotherapy drugs are most damaging to DNA if given to cells at the cell-cycle stage at which DNA replication occurs ${ }^{8}$. It might therefore be expected that cells given chemotherapy during DNA replication would have higher levels of p21 than would cells treated before DNA replication occurs. Yet, surprisingly, Hsu and colleagues found that cells treated during DNA replication had high levels of DNA damage but low levels of p21, and that levels of p21 then increased over time. By contrast, drug treatment before DNA replication resulted in a rapid rise in p21 expression that, depending on the individual cell, either returned to a low level or rose further.

How do some cancer cells that have undergone drug-induced DNA damage revert to having low levels of $\mathrm{p} 21$ expression and gain the ability to divide? The authors propose a model that incorporates dynamic regulation of p21 expression and the level of DNA damage. They suggest that cell fate after chemotherapy shows a property termed bistability - cells are poised to follow one of two fates.

In this scenario, at the cell-cycle stage before DNA replication, if cells express intermediate levels of p21 and small fluctuations occur in signals identifying DNA damage due to chemotherapy, such fluctuations might promote either the rapid induction or decline of p21 expression, driving cells to, respectively, enter senescence or divide.

However, when cells undergo DNA replication, a stage of the cell cycle at which DNA-damage signals are higher than normal (because errors can occur during DNA replication), only a slight increase in the level of p21 would be enough to establish a stable state of high p21 that would lead to senescence. Thus, the Goldilocks zone is defined by the level of $\mathrm{p} 21$ and DNA damage, and determines whether cells divide after chemotherapy. To assess the clinical relevance of this finding, the dose and drug dependency of p21 dynamics during chemotherapy should be examined in detail.

These results raise the possibility that targeting cancer cells at specific cell-cycle stages would produce major differences in the cellular response to chemotherapy, with cells targeted during or shortly after DNA replication being more likely to enter senescence than cells targeted before replication. This model should be investigated further. Taking such an approach in the clinic would pose challenges, however, given that tumours contain a mixture of cell populations that are at different cell-cycle stages. One strategy to tackle this could be to direct cells towards the specific cell-cycle stage at which chemotherapy would be most effective. The authors found that if cells were treated with a small molecule that triggers DNA replication, senescence occurred more commonly than cell division after chemotherapy.

Another challenge will be to identify the optimal outcome for a given cancer following chemotherapy. Although senescence might be an ideal response for certain tumours, others that are more prone to dying in response to cellular damage might be more effectively treated by inducing cell death rather than by triggering senescence.

Hsu and colleagues' work provides a detailed foundation for understanding what governs the fate of cancer cells after chemotherapy. Now it is time to build on this progress, to determine whether strategies can be found that maximize the effectiveness of our current arsenal of anticancer agents.

\section{Yunpeng Liu and Michael T. Hemann \\ are at the Koch Institute for Integrative \\ Cancer Research, Massachusetts Institute of \\ Technology, Cambridge, Massachusetts 02139, USA. \\ e-mails:hemann@mit.edu; yunpengl@mit.edu}

1. Campisi, J. \& d'Adda di Fagagna, F. Nature Rev. Mol. Cell Biol. 8, 729-740 (2007).

2. Campisi, J. Annu. Rev. Physiol. 75, 685-705 (2013).

3. Ewald, J. A., Desotelle, J. A., Wilding, G. \& Jarrard, D. F. J. Natl Cancer Inst. 102, 1536-1546 (2010).

4. Hsu, C.-H., Altschuler, S. J. \& Wu, L. F. Cell 178, 361-373 (2019).

5. d'Adda di Fagagna, F. Nature Rev. Cancer 8, 512-522 (2008).

6. Fitzgerald, A. L. et al. Cell Death Dis. 6, e1678 (2015).

7. Abbas, T. \& Dutta, A. Nature Rev. Cancer 9, 400-414 (2009).

8. Potter, A. J. et al. Carcinogenesis 23, 389-401 (2002).

This article was published online on 5 August 2019. 